\title{
Trade-off analysis approach for interactive nonlinear multiobjective optimization
}

\author{
Petri Eskelinen · Kaisa Miettinen
}

C Springer-Verlag 2011

\begin{abstract}
When solving multiobjective optimization problems, there is typically a decision maker (DM) who is responsible for determining the most preferred Pareto optimal solution based on his preferences. To gain confidence that the decisions to be made are the right ones for the DM, it is important to understand the trade-offs related to different Pareto optimal solutions. We first propose a trade-off analysis approach that can be connected to various multiobjective optimization methods utilizing a certain type of scalarization to produce Pareto optimal solutions. With this approach, the DM can conveniently learn about local trade-offs between the conflicting objectives and judge whether they are acceptable. The approach is based on an idea where the $\mathrm{DM}$ is able to make small changes in the components of a selected Pareto optimal objective vector. The resulting vector is treated as a reference point which is then projected to the tangent hyperplane of the Pareto optimal set located at the Pareto optimal solution selected. The obtained approximate Pareto optimal solutions can be used to study trade-off information. The approach is especially useful when trade-off analysis must be carried out without increasing computation workload. We demonstrate the usage of the approach through an academic example problem.
\end{abstract}

Keywords Multicriteria optimization - Interactive methods · Trade-off rate · Reference point $\cdot$ Pareto optimality $\cdot$ Decision support

\section{Introduction}

In multiobjective optimization problems we are optimizing several conflicting objective functions simultaneously. Because of the conflicting nature of the objectives, we

P. Eskelinen · K. Miettinen $(\bowtie)$

Department of Mathematical Information Technology,

P.O.Box 35 (Agora), 40014 University of Jyväskylä, Finland

e-mail: kaisa.miettinen@jyu.fi 
can identify compromise solutions, so-called Pareto optimal solutions, where we cannot improve the value of any objective function without impairing at least one of the others. A trade-off represents giving up in one of the objectives, which allows the improvement of another objective. Thus, different Pareto optimal solutions involve different trade-offs between the objective functions (see, e.g., Chankong and Haimes 1983; Miettinen 1999). The purpose of multiobjective optimization methods is to offer support and ways to find the best compromise solution. In this, a decision maker (DM) and his preference information play an important role. By a DM we mean a person who is an expert in the domain of the problem considered and who typically is responsible for the final solution.

When considering Pareto optimal solutions, the DM may think how much must one give up of a certain objective in order to improve another one to a certain quantity. Multiobjective optimization methods can be classified based on how the DM is involved in the solution process (see, e.g., Miettinen 1999). We can identify a priori, interactive and a posteriori methods depending on whether the DM specifies preference information before, during or after the solution process, respectively. Especially in the case of interactive and a posteriori methods the DM typically ends up comparing a set of Pareto optimal solutions. In interactive methods the DM iteratively directs the solution process by indicating his preferences related to solution candidates shown. On the other hand, in a posteriori methods the DM selects, in a way or another, the best compromise solution from a generated set of solutions. In both cases the methods usually offer only a set of solutions for the DM to analyze without any additional trade-off information informing the DM about attainable changes in objective function values in the neighborhood of each solution. However, seeing trade-off information between objectives at some given Pareto optimal solution candidate is very important because by definition to gain in the value of some objective function necessitates always tradeoff with the value of some other objective function. The DM benefits from gaining understanding of the interdependencies between the conflicting objectives. From a practical point of view, need for trade-off information is also strongly motivated by the experiences the authors have had with several decision makers who have used the interactive NIMBUS method (see, e.g., Miettinen 1999; Miettinen and Mäkelä 2006) because they have missed some hints indicating what kind of Pareto optimal solutions could be found near the presented ones.

As pointed out, e.g., in Miettinen et al. (2008), we can define both objective and subjective trade-offs. An objective trade-off is based on the structure of the problem and measures the change in one objective in relation to the change in another one, when moving from a feasible solution to another one. On the other hand, a subjective trade-off measures how much the DM considers desirable to sacrifice in the value of some objective function in order to improve another objective to a certain quantity. In what follows, we retain to objective trade-offs. In addition to objective trade-off as characterized above, we can consider objective trade-off rates reflecting infinitesimal change trends when moving from a Pareto optimal solution along a feasible direction. Some examples of interactive methods that utilize objective trade-off rate information include ISWT (Chankong and Haimes 1977), SPOT (Sakawa 1982), STOM (Nakayama and Sawaragi 1984), GRIST (Yang 1999) and IMOOP (Tappeta and Renaud 1999). 
In this paper, we propose a trade-off analysis approach which consists of two distinct parts. The first part is a so-called local trade-off rate analysis tool which can be used to analyze trade-off rate information in the neighborhood of a selected Pareto optimal solution. The second part is a so-called applicability study procedure which can be utilized if the results obtained with the trade-off analysis tool need to be further utilized. A central, important feature of the approach proposed is that it does not necessitate any additional evaluations of the functions of the underlying multiobjective optimization problem. Therefore, it is especially convenient in the case of computationally costly problems where trade-off information would be welcome but, e.g., generating new Pareto optimal solutions would mean waiting times for the DM.

The trade-off analysis tool proposed offers for the DM a way to analyze local trade-off rates at Pareto optimal solutions obtained during the solution process of a multiobjective optimization problem. The term tool is used here to emphasize the fact that our approach is not a standalone method but it is meant to be connected as an additional element into an implementation of an already existing multiobjective optimization method. Whenever a Pareto optimal solution is produced by using a certain type of a scalarization function, we are able to obtain the related local trade-off rate information as a byproduct from the optimal Karush-Kuhn-Tucker multipliers. In principle, this means that when an appropriate numerical solver is used to solve the scalarization problems, we simultaneously also get the multipliers and no additional computation is needed to obtain local trade-off rate information.

Trade-off rate interpretation of the multipliers can be obtained through many different scalarization functions (see, e.g., Sakawa and Yano 1990). However, in this paper we build our trade-off analysis tool around the concept of a reference point consisting of desirable objective function values specified by the DM. Therefore, we assume that in the multiobjective optimization method used, Pareto optimal solutions are produced through optimization of an achievement scalarizing function (see, e.g., Wierzbicki 1986). Despite this limitation, we still have numerous multiobjective optimization methods that can take advantage of the tool. Namely, for instance, many scalarization functions which utilize the Chebyshev norm fit directly into this setting since they can be typically interpreted in the achievement scalarizing function context (of a min-max form). Furthermore, in the case of a posteriori methods, we can also consider such hybrid methods that are based on some evolutionary multiobjective optimization method but where each member of the final population is projected into the set of Pareto optimal solutions using the achievement scalarizing function (see, e.g., Sindhya et al. 2011).

In addition to trade-off rate analysis based on the reference point concept, we propose a supplementary applicability study which can be used to indicate whether the reference point determined by the DM in the trade-off analysis produces a "realistic" approximate Pareto optimal solution. This information is useful when the results of the trade-off analysis tool are used to produce a new Pareto optimal solution.

In what follows, in Sect. 2 we briefly present concepts and notation used. The tradeoff analysis tool is introduced in Sect. 3. In Sect. 4, we propose the applicability study procedure. The usage of the method proposed is demonstrated through an example in Sect. 5. Finally, we conclude in Sect. 6. 


\section{On multiobjective optimization}

We consider nonlinear multiobjective optimization problems of the form

$$
\begin{array}{ll}
\operatorname{minimize} & \left\{f_{1}(\mathbf{x}), \ldots, f_{k}(\mathbf{x})\right\} \\
\text { subject to } & \mathbf{x} \in S
\end{array}
$$

with $k$ conflicting twice-differentiable real-valued objective functions $f_{i}$ which are minimized subject to decision vectors $\mathbf{x}$ belonging to a feasible set $S \subset \mathbb{R}^{n}$. For each $\mathbf{x} \in S$ we can use a mapping $\mathbf{f}: \mathbb{R}^{n} \rightarrow \mathbb{R}^{k}$ to form an attainable objective vector $\mathbf{z}=\mathbf{f}(\mathbf{x})=\left(f_{1}(\mathbf{x}), \ldots, f_{k}(\mathbf{x})\right)^{T}$ belonging to the objective space $\mathbb{R}^{k}$. The image $\mathbf{f}(S) \subseteq \mathbb{R}^{k}$ of the feasible set is called a set of attainable objective vectors.

Next we give some formal definitions for different trade-off terms (see, e.g., Chankong and Haimes 1983). Given two feasible decision vectors $\overline{\mathbf{x}}$ and $\hat{\mathbf{x}}$. We say that

$$
\frac{f_{p}(\overline{\mathbf{x}})-f_{p}(\hat{\mathbf{x}})}{f_{q}(\overline{\mathbf{x}})-f_{q}(\hat{\mathbf{x}})}
$$

is partial trade-off involving $f_{p}$ and $f_{q}$ between $\overline{\mathbf{x}}$ and $\hat{\mathbf{x}}$ if $f_{q}(\overline{\mathbf{x}}) \neq f_{q}(\hat{\mathbf{x}})$ and $f_{i}(\overline{\mathbf{x}})=f_{i}(\hat{\mathbf{x}})$ for all $i=1, \ldots, k$ and $i \neq p, q$. If $f_{i}(\overline{\mathbf{x}}) \neq f_{i}(\hat{\mathbf{x}})$ for some $i=1, \ldots, k$ and $i \neq p, q$ then (2) is called (total) trade-off involving $f_{p}$ and $f_{q}$ between $\overline{\mathbf{x}}$ and $\hat{\mathbf{x}}$. Given feasible decision vector $\overline{\mathbf{x}}$ and direction $\overline{\mathbf{d}} \neq \mathbf{0}$ such that there exists $\bar{\alpha}>0$ so that $\overline{\mathbf{x}}+\alpha \overline{\mathbf{d}} \in S$ for $0 \leq \alpha \leq \bar{\alpha}$. If $f_{i}(\overline{\mathbf{x}})$ is continuously differentiable for each $i=1, \ldots, k$. We say that

$$
\frac{\nabla f_{p}(\overline{\mathbf{x}})^{T} \overline{\mathbf{d}}}{\nabla f_{q}(\overline{\mathbf{x}})^{T} \overline{\mathbf{d}}}
$$

is (total) trade-off rate at $\overline{\mathbf{x}}$ along $\overline{\mathbf{d}}$, when $\nabla f_{q}(\overline{\mathbf{x}})^{T} \overline{\mathbf{d}} \neq 0$. In addition, if there exists $\hat{\alpha}>0$ such that $f_{i}(\overline{\mathbf{x}}+\hat{\alpha} \overline{\mathbf{d}})=f_{i}(\overline{\mathbf{x}})$ for all $i=1, \ldots, k$ and $i \neq p, q$ for all $0 \leq \alpha \leq \hat{\alpha}$ then (3) is called partial trade-off rate.

For the DM, potentially interesting solutions of problem (1) can by identified by using the concept of Pareto optimality. A decision vector $\dot{\mathbf{x}} \in S$ and the corresponding objective vector $\mathbf{f}(\dot{\mathbf{x}})$ are Pareto optimal if there exists no other decision vector $\mathbf{x} \in S$ such that $f_{i}(\mathbf{x}) \leq f_{i}(\dot{\mathbf{x}})$ for all $i=1, \ldots, k$ and at least one of the inequalities is strict. In what follows, we refer to a Pareto optimal decision vector and the corresponding objective vector by the term Pareto optimal solution. If an objective vector is Pareto optimal, we say that it is not dominated by any other attainable objective vector, that is, it is nondominated. In this text, when it is said that an objective vector is dominated or nondominated we mean the dominance in the sense of Pareto optimality. In the objective space, Pareto optimal objective vectors can be identified using a positive orthant cone $\mathbb{R}_{+}^{k}=:\left\{\mathbf{z} \in \mathbb{R}^{k}: z_{i} \geq 0\right.$, for $\left.i=1, \ldots, k\right\}$ as a dominance structure. If $\dot{\mathbf{z}}$ is Pareto optimal then $\left(\dot{\mathbf{z}}-\mathbb{R}_{+}^{k}\right) \cap(\mathbf{f}(S) \backslash\{\dot{\mathbf{z}}\})=\emptyset$. We use a term Pareto surface for the set $P=\mathbf{f}(E)$, where $E \subseteq S$ is the set of Pareto optimal decision vectors of problem (1). A decision vector $\dot{\mathbf{x}} \in S$ is weakly Pareto optimal if there does not exist 
another decision vector $\mathbf{x} \in S$ such that $f_{i}(\mathbf{x})<f_{i}(\dot{\mathbf{x}})$ for all $i=1, \ldots, k$. The set of Pareto optimal decision vectors is always a subset of the set of weakly Pareto optimal decision vectors.

In what follows, we assume that we have a set $P^{\prime} \subset P$ of Pareto optimal objective vectors available for some given multiobjetive optimization problem (1), and we also have an approximate Pareto optimal objective vector $\tilde{\mathbf{z}} \in \mathbb{R}^{k}$. We say that $\tilde{\mathbf{z}}$ is a realistic approximate Pareto optimal solution with respect to $P^{\prime}$, if none of the vectors in set $P^{\prime} \cup\{\tilde{\mathbf{z}}\}$ dominates each other.

In this paper, we concentrate on such interactive and a posteriori multiobjective optimization methods where (weakly) Pareto optimal solutions are computed by minimizing the differentiable formulation of an achievement scalarizing function (see, e.g., Wierzbicki 1986). In other words, we are solving the problem

$$
\begin{array}{ll}
\operatorname{minimize} & \alpha \\
\text { subject to } & w_{i}\left(f_{i}(\mathbf{x})-\overline{\mathbf{z}}\right) \leq \alpha, \quad \text { for } i=1, \ldots, k \\
& \mathbf{x} \in S, \alpha \in \mathbb{R} .
\end{array}
$$

The aspiration levels $\bar{z}_{i}$ indicate desirable levels of the objective functions $f_{i}$, for $i=1, \ldots, k$. These values form a reference point $\overline{\mathbf{z}}=\left(\bar{z}_{1}, \ldots, \bar{z}_{k}\right)^{T}$ and by solving (4) we project the reference point to the Pareto optimal set. For brevity of presentation, we discuss a formulation (4) that generates weakly Pareto optimal solutions. Weak Pareto optimality can be avoided, for instance, by considering an augmented version of (4) (see, e.g., Miettinen 1999).

The scaling coefficients $w_{i}>0$ can be used to determine the projection direction, that is, how the given reference point $\overline{\mathbf{z}}$ is projected to the set of Pareto optimal solutions. One typically used scaling uses ranges, that is, $w_{i}=1 /\left(z_{i}^{\text {up }}-z_{i}^{\text {low }}\right)$, where $z_{i}^{\text {up }}$ and $z_{i}^{\text {low }}$ are approximate (computed or given by the DM) high and low values for the objective function $f_{i}$ attainable in the Pareto optimal set, respectively. In what follows, when we say that $\dot{\mathbf{x}}$ is an optimal solution of (2), we implicitly refer to solution $(\dot{\mathbf{x}}, \dot{\alpha})$ because variable $\alpha$ has only a technical meaning to maintain differentiability (see, e.g., Miettinen 1999).

\section{Interactive trade-off analysis tool}

According to the definition of Pareto optimality, moving from some Pareto optimal solution to another one always necessitates some trade-off in objective function values. Locally this kind of trade-off information can be given in a form of trade-off rates which can be used to approximate how many units the value of some objective function changes if we alter the value of some other objective function by one unit (see, e.g., Chankong and Haimes 1983 for more detailed discussion).

Let us assume the DM uses a multiobjective optimization method where Pareto optimal solutions are produced by solving problem (4). At some point of the solution process, to direct the process or to gain confirmation that some Pareto optimal solution can be considered as the most preferred one, the DM may be interested in knowing how the objective function values are changing in a local neighborhood of 
some particular Pareto optimal solution. If the changes seem to be favorable then, for example, a further search can be concentrated around that particular Pareto optimal solution. From the practical point of view, when this kind of analysis is needed, it is not always necessarily efficient or purposeful to carry out accurate computations to reflect what is happening in the set of Pareto optimal solutions (in particular, if the problem is computationally costly and function evaluations take time). Instead, a computationally inexpensive approximation of the Pareto surface can be used to study trade-offs between the objectives.

In what follows, we first describe how a so-called trade-off plane can be used as a very local linear approximation for the set of Pareto optimal objective vectors at a given Pareto optimal solution. Then we construct the trade-off analysis tool which utilizes the trade-off plane and the reference point concept allowing the DM interactively study trade-off rates related to Pareto optimal solutions computed.

\subsection{Linear approximation}

Let us assume that a Pareto optimal solution $\dot{\mathbf{x}}$ for problem (1) is produced by solving problem (4), and the DM wants to study trade-off rates in a local neighborhood of $\dot{\mathbf{z}}=\mathbf{f}(\dot{\mathbf{x}})$. A tangent hyperplane $T(\dot{\mathbf{z}})$ for the Pareto surface $P$ at $\dot{\mathbf{z}}$ can be used to reflect trade-off rate information. In other words, we locally produce a linear approximation for the nonlinear (possibly also nonconvex) Pareto surface. In what follows, we refer to $T(\dot{\mathbf{z}})$ as a trade-off plane.

For problem (4), using the results presented by Yano and Sakawa (1987) and Sakawa and Yano (1990), the trade-off plane can be obtained from the Karush-Kuhn-Tucker (KKT) multipliers. Namely, if $\dot{\mathbf{x}}$ is a solution of (4) for some $\mathbf{w}$ and $\overline{\mathbf{z}}$, with optimal KKT multipliers $\lambda_{i}$ related to constraints $w_{i}\left(f_{i}(\mathbf{x})-\bar{z}_{i}\right) \leq \alpha$ which are assumed to be active for all $i=1, \ldots, k$, and if in addition at $\dot{\mathbf{x}}$ the following assumptions hold

(i) second-order KKT sufficiency conditions are satisfied,

(ii) the gradients of all active constraints are linearly independent,

(iii) all active constraints have strictly positive KKT multipliers

then the partial trade-off rate involving objectives $f_{1}$ and $f_{i}$ at $\dot{\mathbf{x}}$ is $\partial f_{1}(\dot{\mathbf{x}}) / \partial f_{i}=$ $-\left(\lambda_{i} w_{i}\right) /\left(\lambda_{1} w_{1}\right)$, for $i=2, \ldots, k$.

Let us point out, that assumption (i) above implies that first-order KKT sufficiency conditions hold at $\dot{\mathbf{x}}$ and with assumption (ii) it is guaranteed that multipliers related to active constraints of problem (4) are unique (see, e.g., Nocedal and Wright 2006). Furthermore, it must be emphasized that in problem (4) constraints $w_{i}\left(f_{i}(\mathbf{x})-\bar{z}_{i}\right) \leq \alpha$, for $i=1, \ldots, k$, must be active at $\dot{\mathbf{x}}$.

Partial trade-off rate information can be interpreted as a linear approximation to a relative change in the value of $f_{1}$ when the value of $f_{i}(i=2, \ldots, k)$ is changed by one unit and at the same time all the other objectives $f_{j}(j=2, \ldots, k, j \neq i)$ remain at their current values. This information can be presented in the form of partial trade-off vectors $\mathbf{t}_{1 i}(\dot{\mathbf{x}}) \in \mathbb{R}^{k}$, where the first component is $-\left(\lambda_{i} w_{i}\right) /\left(\lambda_{1} w_{1}\right)$, the $i$ :th component is 1 , and the other components $(j=2, \ldots, k, j \neq i)$ are zero. We can directly conclude that vector $\mathbf{n}=-\left(\lambda_{1} w_{1}, \lambda_{2} w_{2}, \ldots, \lambda_{k} w_{k}\right)^{T}$ is orthogonal to $\mathbf{t}_{1 i}(\dot{\mathbf{x}})$, for all $i=2, \ldots, k$. In other words, we can express a normal vector in an explicit 
form whenever we are able to compute partial trade-off vectors (see, e.g., Yang 1999; Yang and Li 2002).

The normal vector $\mathbf{n}$ for the Pareto surface $P$ at some $\dot{\mathbf{z}} \in P$ can be used to characterize other objective vectors $\mathbf{z} \in T(\dot{\mathbf{z}}) \subset \mathbb{R}^{k}$ in the neighborhood of $\dot{\mathbf{z}}$ in the form $\mathbf{n}^{T}(\mathbf{z}-\dot{\mathbf{z}})=0$. Because the plane $T(\dot{\mathbf{z}})$ is giving only a linear approximation for the Pareto surface, we must point out that in the nonlinear case, a vector $\mathbf{z} \in T(\dot{\mathbf{z}})$ is most likely not Pareto optimal (when $\mathbf{z} \neq \dot{\mathbf{z}}$ ), and it might be even unattainable but it can provide approximate information in the neighborhood of $\dot{\mathbf{z}}$.

In what follows, we assume that KKT multipliers are available as byproducts after having solved problem (4) with an appropriate single objective optimization method. In addition, we restrict our consideration to Pareto optimal solutions of problem (1), where it is possible to numerically compute the trade-off plane $T(\dot{\mathbf{z}})$ using KKT multipliers (see, e.g., Nocedal and Wright 2006, p. 337; Kuk et al. 1997 how the assumptions (i), (ii), and (iii) above can be numerically verified). If at some Pareto optimal solution we are not able to compute the trade-off plane, then the DM can be informed. It must be also pointed out that since our approach can be applied for nonconvex problems, it is assumed that we are in every case able to produce a globally optimal solution for (4). In practice, this might mean that we have to use a separate solver to first reach the area where a locally optimal solution is also globally optimal and after this use some local solver which is producing the final solution and the related KKT multipliers.

\subsection{Trade-off analysis tool}

Let us assume that the DM wants to study the trade-off information behavior in a local neighborhood of a Pareto optimal solution $\dot{\mathbf{z}}$ but unnecessary evaluations of the functions in (1) should be avoided because it is relatively time consuming. The DM is assumed to determine how to change objective values in $\dot{\mathbf{z}}$ to get a more preferred solution. However, instead of using this preference information to generate new Pareto optimal solutions, we use trade-off analysis to study whether the desired changes are reachable. This analysis is computationally inexpensive to carry out because it is based on the trade-off plane information obtained by using the KKT multipliers related to $\dot{\mathbf{z}}$.

In what follows, a vector $\mathbf{d} \in \mathbb{R}^{k}$ (expressed by the DM) is used to indicate desired changes in the objective function values at $\dot{\mathbf{z}}$. This determines a reference point $\overline{\mathbf{z}}=(\dot{\mathbf{z}}+$ d) which can be used in a linear achievement scalarizing problem where the task is to

$$
\begin{aligned}
\operatorname{minimize} & \alpha \\
\text { subject to } & w_{i}\left(z_{i}-\left(\dot{z}_{i}+d_{i}\right)\right) \leq \alpha, \quad \text { for } i=1, \ldots, k \\
& \mathbf{n}^{T}(\mathbf{z}-\dot{\mathbf{z}})=0 \\
& \mathbf{z} \in \mathbb{R}^{k}, \alpha \in \mathbb{R} .
\end{aligned}
$$

In other words, in (5), the Pareto surface is approximated linearly at $\dot{\mathbf{z}}$ (see Fig. 1) and the solution which is in a sense closest to the new reference point located on this trade-off plane is found (in Fig. 1 this solution is denoted by $\tilde{\mathbf{z}}$ ) and shown to the DM. This solution is called an approximate Pareto optimal solution and it is supposed to give some insight for the DM about the reachability of the desired changes expressed. 


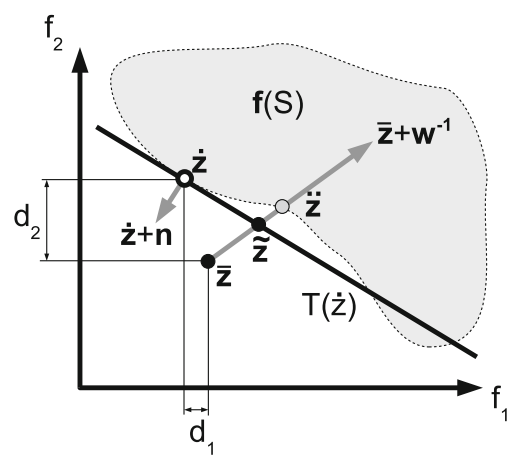

Fig. 1 Approximation of the Pareto surface

Because the approximate solution corresponding to the new reference point is obtained basically very fast (by solving a linear problem), the DM can judge in real-time the kind of trade-offs available in a local neighborhood of $\dot{\mathbf{z}}$ and give different desirable changes, if needed. For example, if trade-offs related to $\dot{\mathbf{z}}$ seem to be promising, it is always possible to solve problem (4) corresponding to the reference point $\overline{\mathbf{z}}$ to obtain a new actually attainable Pareto optimal solution (but this may take some time with computationally expensive problems).

It may be preferable, even though not necessary, that the changes in $\mathbf{d}$ are set in a way that obeys the principle of Pareto optimality, that is, if the value of some objective is to be improved then the value of at least one objective should be relaxed. This is an important issue because if the preferences are set according to the principle of Pareto optimality, it is more likely that the DM can experience that the tool is able to produce Pareto optimal solutions which correspond to preferences expressed (feeling of control). Problem (5) has a unique solution except if the normal vector $\mathbf{n}$ is parallel to some of the coordinate axes in the objective space $\mathbb{R}^{k}$.

For our purposes, it is enough to use information that the projection direction in problem (4) is determined by a vector $\mathbf{w}^{-1}=\left(1 / w_{1}, \ldots, 1 / w_{k}\right)$, where $w_{i}>0$ $(i=1, \ldots, k)$ are the scaling coefficients. Thus, instead of actually solving the linear problem (5), we can directly compute where the projection direction vector $(\dot{\mathbf{z}}+\mathbf{d}+$ $\left.t \mathbf{w}^{-1}\right)$ and trade-off plane $\mathbf{n}^{T}(\mathbf{z}-\dot{\mathbf{z}})=0$ are intersecting. This means solving for $t$ (to be denoted by $\tilde{t})$ in equation $\mathbf{n}^{T}\left(\left(\dot{\mathbf{z}}+\mathbf{d}+t \mathbf{w}^{-1}\right)-\dot{\mathbf{z}}\right)=0$ which we can write in the form $\tilde{t}=-\left(\mathbf{n}^{T} \mathbf{d}\right) /\left(\mathbf{n}^{T} \mathbf{w}^{-1}\right)$, where $\mathbf{n}^{T} \mathbf{w}^{-1}>0$ because $w_{i}^{-1}>0$ and $n_{i}<0$, for $i=1, \ldots, k$. Then, the approximate Pareto optimal objective vector obtained using the preference information given by the DM is $\tilde{\mathbf{z}}=(\dot{\mathbf{z}}+\mathbf{d})+\tilde{t} \mathbf{w}^{-1}=\overline{\mathbf{z}}+\tilde{t} \mathbf{w}^{-1}$. In this way, the new approximate solution can be obtained even faster than by solving problem (5).

In practice, it is convenient to assume $z_{i}^{\text {low }} \leq \bar{z}_{i} \leq z_{i}^{\text {up }}$ and $z_{i}^{\text {low }} \leq \tilde{z}_{i} \leq z_{i}^{\text {up }}$, for $i=1, \ldots, k$. However, in the above calculation, it happen that $\tilde{z}_{i} \geq z_{i}^{\text {up }}$, for more than one $i=1, \ldots, k$, even though $z_{i}^{\text {low }} \leq \bar{z}_{i} \leq z_{i}^{\text {up }}$, for $i=1, \ldots, k$. This special case can be taken into account but for the sake of clarity we omit such details in this paper.

Figures 1 and 2 demonstrate a situation where the trade-off analysis is carried out at the Pareto optimal solution $\dot{\mathbf{z}} \in P$. In the figures, we have only two objectives to enable 


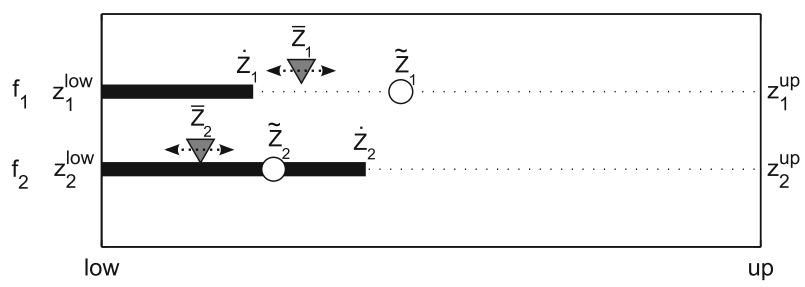

Fig. 2 The selected solution

intuitive visualization. However, it must be emphasized that the trade-off analysis tool can be utilized for multiobjective problems having any number of objectives.

In Fig. 1, we demonstrate the trade-off analysis tool with a simple example, where the DM considers the solution $\dot{\mathbf{z}}$ and is interested in decreasing $f_{2}$ value by allowing $f_{1}$ to increase as indicated by the reference point $\overline{\mathbf{z}}=(\dot{\mathbf{z}}+\mathbf{d})$, where $\mathbf{d}=\left(d_{1}, d_{2}\right)^{T}$ reflects the desired changes. According to the trade-off analysis tool, the approximate solution reflecting $\overline{\mathbf{z}}$ is $\tilde{\mathbf{z}} \in T(\dot{\mathbf{z}})$. The vector $\ddot{\mathbf{z}}$ is the corresponding attainable Pareto optimal objective vector which could be obtained by solving problem (4).

Figure 2 corresponds to the setting depicted in Fig. 2 and it presents a possible user interface for the trade-off analysis tool described above. In the figure, the DM has selected for trade-off analysis the Pareto optimal solution $\dot{\mathbf{z}}$ for which objective function values are visualized using a bar chart (black bars). The triangle marker is a reference point $\overline{\mathbf{z}}=(\dot{\mathbf{z}}+\mathbf{d})$ determined by the DM. The circle marker indicates the approximate Pareto optimal objective vector $\tilde{\mathbf{z}} \in T(\dot{\mathbf{z}})$ obtained using the trade-off analysis tool.

The DM can rapidly experiment with different $\overline{\mathbf{z}}$ 's by altering the reference point and the approximate Pareto optimal objective vector $\tilde{\mathbf{z}}$ is updated accordingly with minimal effort. In this way, the DM can in real-time get an idea of what kind of tradeoffs are available and how his/her preferences correspond to Pareto optimal solutions in the local neighborhood of the selected Pareto optimal solution $\dot{\mathbf{z}}$. The interface proposed in Fig. 2 can be, for instance, implemented in such a way that the DM is able to alter the reference point (triangle markers) rapidly using the mouse.

As an example, let us point out that the trade-off analysis tool can be easily included, for example, as a part of the IND-NIMBUS software (Miettinen 2006) implementing the NIMBUS method discussed in the introduction. This is possible since scaralization (4) is available in NIMBUS. Furthermore, interface depicted in Fig. 2 is very similar to what is available in the IND-NIMBUS software.

After having studied the local trade-off rate information around some Pareto optimal solution $\dot{\mathbf{z}}$ with the trade-off analysis tool, the DM has a couple of options. Naturally, the DM can continue to do the trade-off analysis at some other Pareto optimal solution and in this way implicitly gain insight about the shape of the Pareto surface. On the other hand, if the analysis reveals preferable trade-offs around some particular Pareto optimal solution, then more Pareto optimal solutions can be generated around this solution. How the generation of these new Pareto optimal solutions is carried out depends on the underlying multiobjective optimization method. The most direct approach to continue after the trade-off analysis is to use the reference point $\overline{\mathbf{z}}$ and project it directly to the set of attainable Pareto optimal solutions by solving problem 
(4) (in which case $\tilde{\mathbf{z}}$ gave an idea of what may be the corresponding objective vector values). Especially in interactive methods such as NIMBUS, this option is very appealing. However, also in a posteriori methods some individual Pareto optimal solution can be refined by using our approach.

Whenever $\tilde{\mathbf{z}}$ is relatively far from $\dot{\mathbf{z}}$, we are not able to say how good the approximation really is because $T(\dot{\mathbf{z}})$ gives only a linear approximation. Therefore, in what follows, we propose a supplementary applicability study which can be used to reveal whether $\tilde{\mathbf{z}}$ can be considered as a reliable approximation for some attainable Pareto optimal objective vector.

\section{Applicability study}

The design goal of the applicability study to be proposed is similar to the trade-off analysis tool presented in the previous section. To be more specific, we build up a procedure to provide information about the reliability of an approximation $\tilde{\mathbf{z}} \in T(\dot{\mathbf{z}})$ without evaluating the functions of multiobjective optimization problem (1). Based on the motivation behind the trade-off analysis, it is assumed that the DM is primarily interested in Pareto optimal solutions which have the same trade-off rates as those related to $\tilde{\mathbf{z}}$, which was induced by the reference point $\overline{\mathbf{z}}$. We are referring to the approximate Pareto optimal objective vectors $(\dot{\mathbf{z}}+t(\tilde{\mathbf{z}}-\dot{\mathbf{z}}))$, for $t \in[0,1]$.

Let us assume that when the trade-off analysis takes place, the set $P^{\prime} \subset P$ contains all the Pareto optimal solutions available at that moment. We recall that an approximate Pareto optimal objective vector $\tilde{\mathbf{z}}$ is called realistic with respect to $P^{\prime}$ if none of the vectors in the set $P^{\prime} \cup\{\tilde{\mathbf{z}}\}$ dominates each other. Now, the aim of the applicability study is to indicate if $\tilde{\mathbf{z}}$ related to the reference point $\overline{\mathbf{z}}$ (determined by the DM) is realistic with respect to $P^{\prime}$, and if not, to determine the largest possible $t^{\prime} \in[0,1]$ in such a way that $(\dot{\mathbf{z}}+t(\tilde{\mathbf{z}}-\dot{\mathbf{z}}))$ is realistic for each $t \in\left[0, t^{\prime}\right]$. Furthermore, we say that a Pareto optimal objective vector $\mathbf{z} \in P^{\prime}$ is active if it either dominates or is dominated by $(\dot{\mathbf{z}}+t(\tilde{\mathbf{z}}-\dot{\mathbf{z}}))$, for some $t \in[0,1]$. In other words, the applicability study points out the first Pareto optimal objective vector in $P^{\prime}$ which becomes active when we move from $\dot{\mathbf{z}}$ to $\tilde{\mathbf{z}}$ along the line $(\dot{\mathbf{z}}+t(\tilde{\mathbf{z}}-\dot{\mathbf{z}}))$ where the trade-off rates remain unchanged. Algorithm 1 describes the applicability study in a formal way.

The first phase of Algorithm 1 reveals whether $\dot{\mathbf{z}}+t(\tilde{\mathbf{z}}-\dot{\mathbf{z}})$ dominates any Pareto optimal objective vector in $P^{\prime \prime}$ for some $t \in[0,1]$ and the second one whether $\dot{\mathbf{z}}+t(\tilde{\mathbf{z}}-\dot{\mathbf{z}})$ is dominated by any Pareto optimal objective vectors in $P^{\prime \prime}$. If at the end $\mathbf{z}^{\prime} \neq \dot{\mathbf{z}}$ then $\mathbf{z}^{\prime}$ is the Pareto optimal objective vector which becomes first active when we move from $\dot{\mathbf{z}}$ to $\tilde{\mathbf{z}}$. The example in the next section gives an idea of how the applicability study can be utilized in practice.

It is clear that when we have only very few Pareto optimal solutions available (or if we have a large number of objectives), the applicability study proposed does not necessarily help that much to evaluate whether approximate Pareto optimal solutions obtained with the trade-off analysis tool are realistic or not (this is due to the fact that the objective vectors considered do not dominate each other). In other words, the DM can determine very large variations to the Pareto optimal objective vector selected without being able to say anything about the reliability of the approximation 


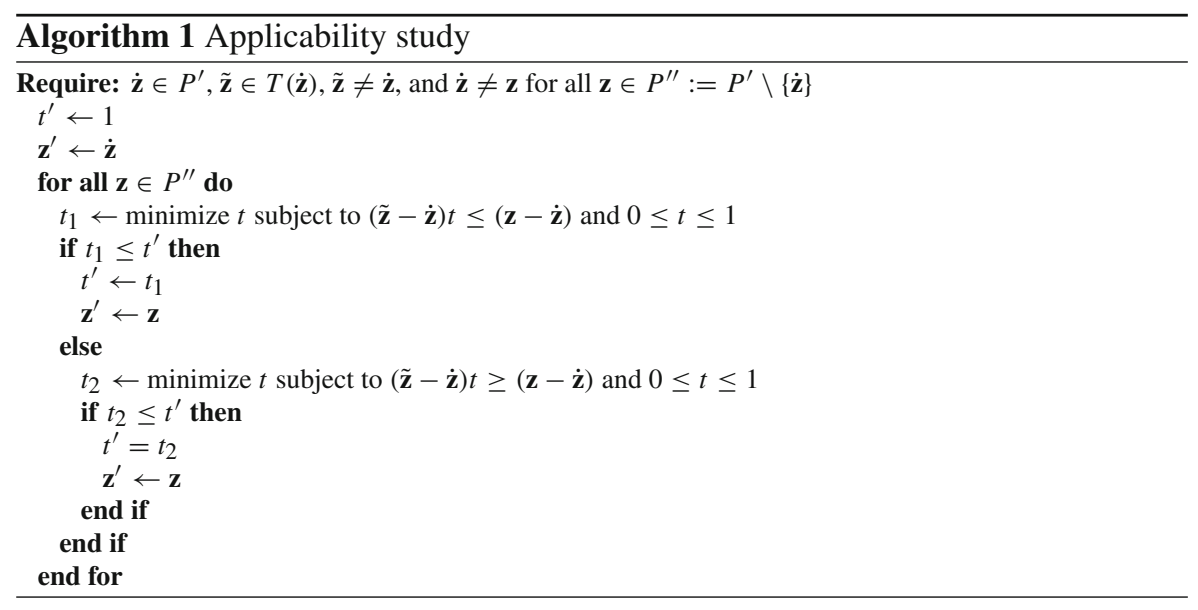

obtained. However, the applicability study presented in this section can be regarded as an addition to the trade-off analysis tool rather than an accurate applicability indicator. The idea is just to take advantage of all information available, that is, the computed Pareto optimal solutions no matter how many they are. Naturally, the more Pareto optimal solutions are available, the better the applicability study is able to identify the approximations which are not realistic or not-and all this without adding the computational cost.

\section{Example}

In this section, we demonstrate the usage of the trade-off analysis tool by considering an academic nonconvex problem having three objective functions. Even though the example problem does not contain any computationally demanding functions, it must be pointed out that while carrying out the trade-off analysis, we do not need to evaluate functions of the original problem.

In this example, the objective functions $f_{i}: \mathbb{R}^{2} \rightarrow \mathbb{R}(i=1,2,3)$ are constructed using function $\phi\left(x_{1}, x_{2}\right)=\psi\left(x_{1}, x_{2}\right)-\exp \left(-50 \psi\left(x_{1}, x_{2}\right)\right)$, where we have $\psi\left(x_{1}, x_{2}\right)=x_{1}^{2}+x_{2}^{2}$. The actual unconstrained problem is expressed in the form

$$
\operatorname{minimize}\left\{f_{1}=\phi\left(x_{1}, x_{2}\right), f_{2}=\phi\left(x_{1}, x_{2}-1\right), f_{3}=\phi\left(x_{1}-1, x_{2}\right)\right\} \text {. }
$$

Let us assume that we have obtained six Pareto optimal solutions using some multiobjective optimization method utilizing problem (4) with different preferences specified by the DM and the DM is now willing to use the trade-off analysis tool to study these solutions. The objective function values of these six solutions are shown in Fig. 3 together with upper and lower bounds for the objective functions. In this case, the DM has determined bounds $\mathbf{z}^{\text {up }}$ and $\mathbf{z}^{\text {low }}$ using his expertise. The Pareto surface and the six Pareto optimal solutions are visualized in Fig. 4 solutions. Even though the Pareto surface is visualized in Fig. 4 its explicit form is assumed to be unknown. 


\begin{tabular}{l|rrr} 
& $f_{1}$ & $f_{2}$ & $f_{3}$ \\
\hline $\mathbf{z}^{\text {low }}$ & -1.00 & -1.00 & -1.00 \\
$\mathbf{z}^{\text {up }}$ & 1.00 & 2.00 & 2.00 \\
\hline$\dot{\mathbf{z}}^{1}$ & -0.18 & 0.78 & 0.78 \\
$\dot{\mathbf{z}}^{2}$ & 0.16 & 0.41 & 0.91 \\
$\dot{\mathbf{z}}^{3}$ & 0.47 & 0.12 & 1.22 \\
$\dot{\mathbf{z}}^{4}$ & 0.16 & 0.91 & 0.41 \\
$\dot{\mathbf{z}}^{5}$ & $\mathbf{0 . 2 8}$ & $\mathbf{0 . 5 3}$ & $\mathbf{0 . 5 3}$ \\
$\dot{\mathbf{z}}^{6}$ & 0.41 & 1.16 & 0.16
\end{tabular}

Fig. 3 Pareto optimal solutions and ranges

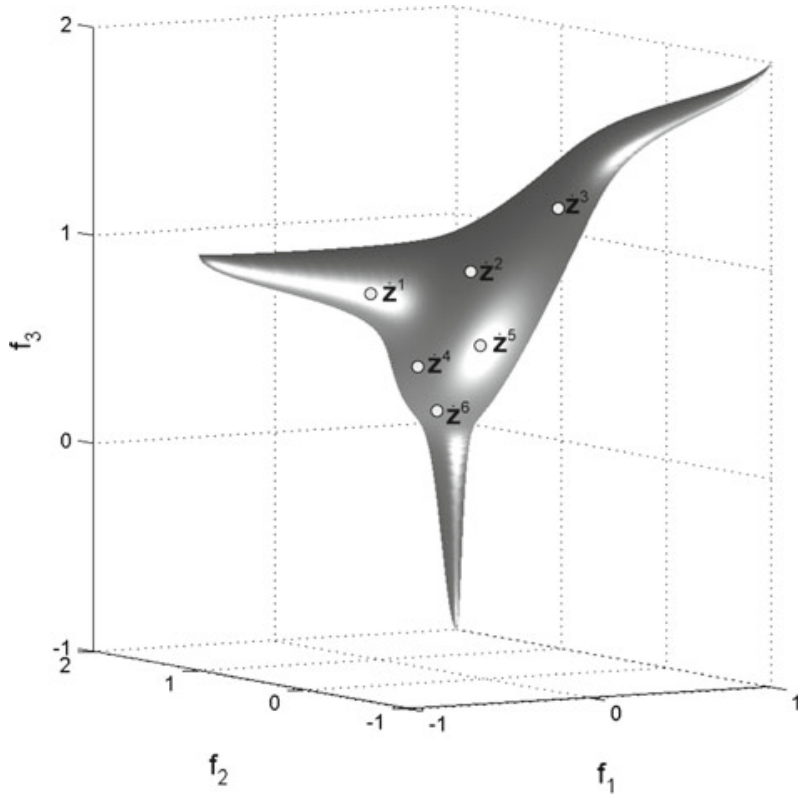

Fig. 4 Set of attainable objective vectors and the six computed Pareto optimal solutions

As already explained in Sect. 3, the DM can change the components of the Pareto optimal objective vector selected to produce a reference point which is then projected to the trade-off plane. Let us assume that we have a simple user interface containing a bar chart visualization for each Pareto optimal objective vector available and the DM has selected $\dot{\mathbf{z}}^{5}$ for the trade-off analysis (the upper part of Fig. 5). In case (a) of Fig. 5 , the DM is interested in studying what kind of solutions are approximately available if he wants to improve the value of $f_{1}$ around $5 \%$ and value of $f_{3}$ around $10 \%$ from $\dot{z}_{1}^{5}$ and $\dot{z}_{3}^{5}$, respectively (percentages refer to ranges $\left.\left(z_{i}^{\text {up }}-z_{i}^{\text {low }}\right), i=1,2,3\right)$. To compensate this improvement, the DM is willing to sacrifice around $5 \%$ in the value of $f_{2}$ from $\dot{z}_{2}^{5}$. The DM proposes the resulting new reference point by adjusting the positions of gray triangle markers appearing in (a) bar chart in Fig. 5. The resulting approximate Pareto 


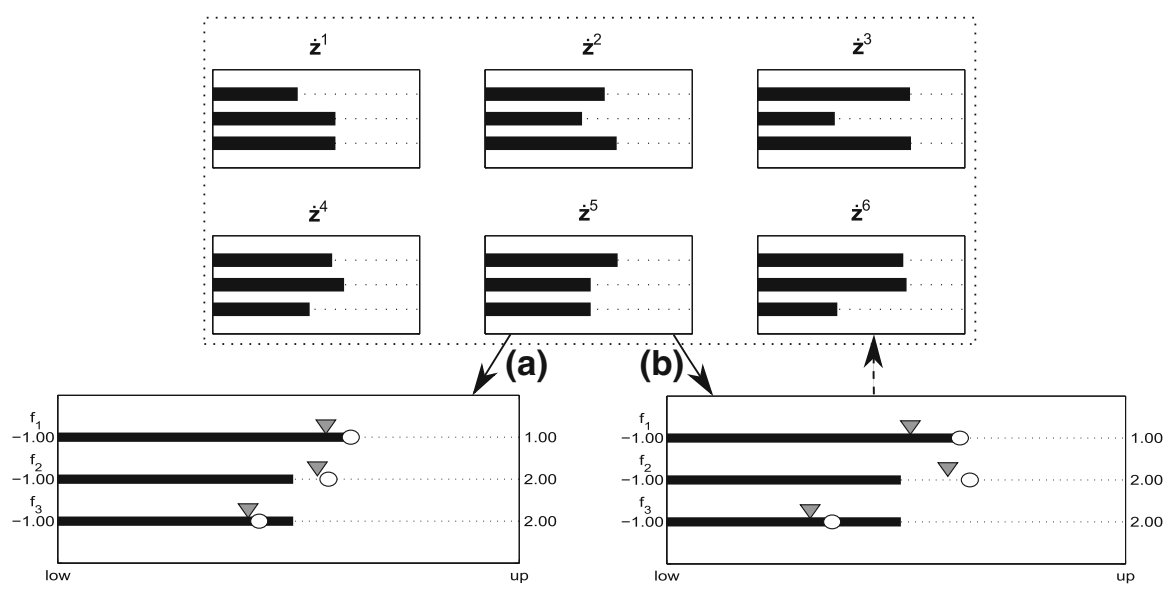

Fig. 5 Trade-off analysis with applicability study: a with realistic and $\mathbf{b}$ with unrealistic approximate solution

optimal objective vector is $\tilde{\mathbf{z}}=(0.27,0.76,0.31)^{T}$ and it is depicted in Fig. 5 with white circles. By using Algorithm 1 we can confirm that the approximation $\tilde{\mathbf{z}}$ can be considered realistic in comparison to the five other Pareto optimal solutions available. However, we do not know how far from the Pareto surface $P$ the approximate Pareto optimal objective vector $\tilde{\mathbf{z}}$ really is.

Case (b) of Fig. 5 illustrates the situation where the DM has proposed twice as large changes to get a new reference point as in case (a) and the trade-off rates between objective functions remain, therefore, unchanged. However, now the reference point $\overline{\mathbf{z}}$ produces the approximate Pareto optimal objective vector $\tilde{\mathbf{z}}=(0.27,0.96,0.08)^{T}$ which dominates the Pareto optimal objective vector $\dot{\mathbf{z}}^{6}$ and, thus, the approximation regarded not to be realistic. Because the approximation obtained is not realistic, the applicability study suggests to the DM that maybe he would like to carry out trade-off analysis based on the Pareto optimal solution $\dot{\mathbf{z}}^{6}$ instead of the current solution $\dot{\mathbf{z}}^{5}$. However, it must be emphasized that this is only a suggestion and the DM is allowed to continue the analysis even though the resulting approximation is not realistic. The approximate Pareto optimal vectors can be used by the DM to study the local tradeoff rate information around the selected Pareto optimal solution and the result of the applicability study can be considered only as a warning.

\section{Conclusions}

We have introduced a trade-off analysis approach which can be integrated into any implementation of an interactive or a posteriori multiobjective optimization method utilizing an achievement scalarizing function an equivalent scalarization formulation for generating Pareto optimal solutions. The approach provides a possibility to make the most of information generated as a byproduct of solution generation. The approach has two distinct parts. The first part is a local trade-off analysis tool, where trade-off rates can be studied by projecting a reference point into a linear approximation of the set of Pareto optimal objective vectors at a given Pareto optimal solution. The tool utilizes 
the well-known trade-off rate interpretation of the Karush-Kuhn-Tucker multipliers. Since the multipliers can be obtained as a byproduct when a Pareto optimal solution is generated by solving the achievement scalarizing problem with an appropriate solver, the computational burden caused by this trade-off analysis is minimal. Thus, the tool is particularly useful for supporting DMs dealing with computationally expensive problems where function evaluations take time. With the tool, the DM can judge the feasibility of one's preferences without solving the actual time-consuming problem.

The second part of the approach proposes a supplementary applicability study which can be used to analyze whether the reference point used in the trade-off analysis can be considered to produce a reliable approximate Pareto optimal solution. Also this applicability study utilizes only the information available at the moment when the trade-off analysis takes place. Therefore, the fundamental idea behind the approach presented is that additional function evaluations related to the multiobjective optimization problem are completely avoided and the information available is utilized as widely as possible. We have also demonstrated the usage of the approach with a simple example.

\section{References}

Chankong V, Haimes YY (1977) The interactive surrogate worth trade-off (ISWT) method for multiobjective decision making. In: Zionts S (ed) Multiple criteria problem solving, vol 155. Springer, Berlin, pp 42-67

Chankong V, Haimes YY (1983) Multiobjective decision making theory and methodology. Elsevier Science Publishing Co., Inc., New York

Kuk H, Tanino T, Tanaka M (1997) Trade-off analysis for vector optimization problems via scalarization. J Inf Optim Sci 18:75-87

Miettinen K (1999) Nonlinear multiobjective optimization. Kluwer, Boston

Miettinen K (2006) IND-NIMBUS for demanding interactive multiobjective optimization. In: Trzaskalik T (ed) Multiple criteria decision making '05. The Karol Adamiecki University of Economics in Katowice, Katowice, pp 137-150

Miettinen K, Mäkelä MM (2006) Synchronous approach in interactive multiobjective optimization. Eur J Oper Res 170(3):909-922

Miettinen K, Ruiz F, Wierzbicki AP (2008) Introduction to multiobjective optimization: interactive approaches. In: Branke J, Deb K, Miettinen K, Slowinski R (eds) Multiobjective optimization: interactive and evolutionary approaches. Springer, Berlin, pp 27-57

Nakayama H, Sawaragi Y (1984) Satisficing trade-off method for multiobjective programming. In: Grauer M, Wierzbicki AP (eds) Interactive decision analysis. Springer, New York, pp 113-122

Nocedal J, Wright SJ (2006) Numerical Optimization, 2nd edn. Springer, New York

Sakawa M (1982) Interactive multiobjective decision making by the sequential proxy optimization technique: SPOT. Eur J Oper Res 9:386-396

Sakawa M, Yano H (1990) Trade-off rates in the hyperplane method for multiobjective optimization problems. Eur J Oper Res 44:105-118

Sindhya K, Deb K, Miettinen K (2011) Improving convergence of evolutionary multi-objective optimization with local search: a concurrent hybrid algorithm. Nat Comput. doi:10.1007/s11047-011-9250-4

Tappeta RV, Renaud JE (1999) Interactive multiobjective optimization procedure. AIAA J 37:881-889

Wierzbicki AP (1986) On completeness and constructiveness of parametric characterizations to vector optimization problems. OR Spektrum 8:73-87

Yang J-B (1999) Gradient projection and local region search for multiobjective optimisation. Eur J Oper Res 112:432-459

Yang J-B, Li D (2002) Normal vector identification and interactive tradeoff analysis using minimax formulation in multiobjective optimization. IEEE Trans Syst Man Cybern Part A: Syst Hum 32(3):305-319

Yano H, Sakawa M (1987) Trade-off rates in the weighted Tchebycheff norm method. Large Scale Syst 13:167-177 